\title{
Materiais de ensino da leitura e o paradigma Psicolinguística e interfaces
}

\author{
Vera Wannmacher Pereira \\ PUCRS
}

\begin{abstract}
Resumo
O trabalho tem como eixo materiais de ensino da leitura nos anos iniciais e formação inicial dos professores. Primeiramente, analisa o aprendizado da leitura. Após, examina as relações entre dificuldades de construção de um paradigma produtivo de materiais de ensino da leitura e formação inicial dos professores. A seguir, propõe um paradigma baseado na Psicolinguística em interface interna com a Linguística do Texto e externa com as Neurociências, a Psicologia Cognitiva e a Educação. Por último, apresenta encaminhamentos para um paradigma de academia com interface de campos do conhecimento, propondo aproximação entre disciplinas de fundamentos e de ensino, diálogo teoria/prática e integração academia/escola.
\end{abstract}

Palavras-chave: materiais de ensino da leitura; anos iniciais; formação inicial de professores; paradigma Psicolinguística e interfaces.

\begin{abstract}
The core of this paper is materials for the teaching of reading, in elementary school and in initial teacher formation. First, it considers the learning of reading. Second, it analyzes the relationship between the difficulties in constructing a productive paradigm of the materials for teaching reading and teacher formation. Following this, it proposes a paradigm based on Psycholinguistics interfaced with Text Linguistics and also Neurosciences, Cognitive Psychology and Education. Finally, it presents directions for an academic paradigm with interface in different knowledge fields. It also proposes a closer relationship between theoretical disciplines and teaching disciplines, a dialogue between theory and practice, and an integration between academia and school.

Keywords: teaching reading materials; elementary school; initial teacher formation; Psycholinguistics and interfaces paradigm.
\end{abstract}

\section{COMENTÁRIOS INICIAIS}

Todos que de alguma forma estão próximos de estudantes dos anos iniciais reconhecem suas dificuldades em compreender textos mesmo muito simples que exigem inferências pouco complexas.

Esse reconhecimento tem provocado desconfortos diversos: familiares assustados com a demora na alfabetização; crianças assaltadas pelo medo do fracasso; professores confusos entre livros didáticos do mercado editorial, determinações 
programáticas das escolas, orientações teóricas da academia e sua própria história como aprendizes; poder público frustrado pelo insucesso de suas iniciativas; academia perplexa diante da correlação negativa entre o esforço de ensino realizado e o benefício de aprendizado alcançado.

Com o objetivo de contribuir para o entendimento dessa situação e, se possível, para sua superação, o presente estudo elege como eixo materiais de ensino da leitura, no contexto da academia, para estudantes que frequentam os anos iniciais. Nessa escolha, os professores universitários, os seus alunos e os futuros alunos desses estão entrelaçados desempenhando importantes papéis na construção de novos paradigmas em que os materiais de ensino possam ter especial relevância.

Para isso, neste ensaio, é feita uma análise dessa situação, a seguir, é proposto um paradigma possível para trabalho com a leitura, com apoio na Psicolinguística e interfaces, e, por último, são sugeridos caminhos para a academia como segmento responsável pela formação inicial dos professores.

\section{ANÁLISE DA SITUAÇÃo}

Uma reflexão sobre as dificuldades para mudança de paradigmas no ensino da língua materna conduz ao levantamento dos possíveis motivos que as constituíram.

Alguns desses motivos são conferidos ao professor - conhecimentos teóricos fragmentados, despreparo metodológico, universo de histórias e crenças vinculado a antigos paradigmas. Outros são atribuídos às instituições formadoras - multiplicidade de caminhos teóricos não convergentes disponibilizados nas licenciaturas e nas iniciativas extensionistas de capacitação e ausência de orientações para transposições didáticas. Outros ainda estão vinculados aos alunos e seus familiares - disposição fraca para os estudos escolares e resistência a novos caminhos de ensino. Outros mais estão associados aos órgãos mantenedores - orientação escassa e acompanhamento insuficiente do professor como pessoa e como profissional.

Desse conjunto de possibilidades, a análise aqui recai sobre a formação acadêmica inicial do professor de língua materna, com foco em materiais de ensino da leitura para estudantes dos anos iniciais.

Esse professor de língua materna por muito tempo foi visto exclusivamente como o licenciado em Letras com concentração na Língua Portuguesa. Ao professor com Curso de Magistério (hoje com Curso de Pedagogia, por exigência de legislação) 
foi reconhecida a responsabilidade de ensinar conteúdos de língua materna, mas não de disciplina específica de Língua Portuguesa. Do mesmo modo, ao licenciado em Letras não foi concedido o direito de trabalho com anos iniciais.

Mais recentemente, vem ocorrendo uma mudança importante em relação a essas atribuições. Dadas às imensas dificuldades de aprendizado da leitura e da escrita, os linguistas têm se associado às preocupações de todos e contribuído com seus conhecimentos para análise e solução do problema. Esse novo posicionamento está gerando uma forte tendência para os trabalhos de interfaces, o que vem sendo produtivo, por permitir aclaramentos desse problema sob diversos ângulos. Neste artigo, a base teórica provém da Psicolinguística em interface interna com a Linguística do Texto e em interface externa com as Neurociências, a Psicologia Cognitiva e a Educação.

Essa mudança está tendo como resultado o fato de que o Curso de Pedagogia e o Curso de Letras têm voltado seu olhar para o ensino da língua materna nos anos iniciais. Como decorrência, disciplinas que procuram explicitar os processos de aquisição e aprendizado da língua estão presentes nos dois cursos. Além disso, por decorrência de interesses dos professores de Linguística do Curso de Letras, os acadêmicos participam ao longo de sua formação de pesquisas que focalizam os anos iniciais. É desse lugar que a autora deste artigo, linguista comprometida com a academia e com a escola e com histórico considerável de docência nesses dois segmentos, desenvolve análises e propõe encaminhamentos com base em sua área de estudo - a leitura sob a perspectiva da Psicolinguística e suas interfaces.

Nesses cursos, há a preocupação com o desenvolvimento de conhecimentos linguísticos teóricos e com sua aplicação em materiais de ensino, evidenciando-se com nitidez uma ênfase teórica em Letras e uma ênfase prática na Pedagogia. No entanto, tanto numa como noutra situação há um certo vazio a ser preenchido para que o acadêmico adquira o necessário preparo para a construção de materiais de ensino da língua materna capazes de contribuir para a modificação de paradigmas em relação a esse aspecto. É importante sublinhar que esse preparo significa não só conhecer os princípios teóricos e metodológicos, mas examinar materiais de ensino já elaborados.

Nessa análise, cabe primeiramente uma reflexão sobre um pensamento que muitas vezes domina os estudos sobre as dificuldades para mudar paradigmas no ensino da língua materna. Esse pensamento situa no professor a causa primeira, atribuindo-lhe resistência a mudanças. 
É um tanto intrigante o fato de que nas aulas de História, Geografia, Ciências, Matemática não é incomum o desenvolvimento de conhecimentos superados por novas descobertas, enquanto, nas de língua materna, as novas descobertas da Linguística pouco são consideradas. Dificilmente não estão sendo discutidas atualmente nas aulas as novas teorias sobre as movimentações do planeta Terra, os conflitos sociais no Oriente, ou a emoção no cérebro, no entanto, embora todas as evidências de que a definição de verbo como ação não se sustente, ela persiste nas aulas de língua materna.

Há que se considerar, entretanto, que a natureza das disciplinas primeiramente referidas é nitidamente diferente da dos estudos sobre a língua materna, uma vez que estes examinam um conhecimento decorrente de um processo de aquisição gradativo e impregnado das condições sociais e culturais dos alunos. Evidentemente essa distinção não responde pela diferença de recortes dos conhecimentos, nem a justifica, mas sugere variáveis a levar em conta.

Também há que se considerar que o ensino de língua materna tem absorvido propostas de inovação que têm surgido de diferentes áreas do conhecimento. Cabe examinar essas apropriações numa linha de tempo e refletir sobre o acolhimento pelos professores e respectivos reflexos nos materiais de ensino.

Até 1970, o ensino de língua materna era determinado pelas Secretarias de Educação. Para o então Curso Primário do RS, havia um programa de ensino de "Linguagem", definido por objetivos, conteúdos programáticos e sugestões de atividades, obrigatoriamente aplicado por todas as escolas. À Secretaria de Educação cabia orientar os professores, supervisionar o ensino e avaliá-lo por meio de provas por ela elaboradas. Até esse período as cartilhas e os livros didáticos eram construídos de acordo com esses programas de ensino.

Com a Lei 5692/71, houve a definição de novos programas de ensino não mais seriados, mas com comportamentos de saída para as séries iniciais e para as séries finais do $1^{\circ}$ grau. A Língua Portuguesa passou a ser denominada Língua Nacional e foi diluída na área de Comunicação e Expressão que abrigava igualmente a Educação Artística e a Educação Física. Para dar sustentação à nova ideia, que se baseava na Teoria da Comunicação, foram desenvolvidos cursos de reciclagem dos professores. A partir de então as provas avaliativas passaram ao encargo dos docentes, os encontros de orientação se tornaram muito raros e a supervisão ficou implantada dentro das escolas. Esse novo sistema gerou certa equivalência de conhecimentos entre o supervisor e o professor, o que, contrariamente ao esperado, de certo modo brecou a formação de uma 
massa crítica na escola. É interessante registrar que houve empenho dos professores em cumprir as novas orientações, sendo que a ausência de êxito não pode ser atribuída à falta de adesão dos professores, mas possivelmente aos limites da proposta teórica e às dificuldades em transformá-la em situações de ensino, em materiais de ensino.

Seguindo a linha de tempo, no final dos anos 70, surgiram as primeiras publicações de Emília Ferreiro dentro do ponto de vista de que a construção do conhecimento pela criança é ativo. Investigou as etapas de construção da escrita da palavra cujos resultados foram amplamente utilizados para a elaboração de uma proposta de alfabetização que, por decorrência, situou na escrita o eixo desse processo. A pesquisadora desenvolveu, com apoio em seus estudos, uma cruzada, como ela própria denominou, contra as cartilhas até então utilizadas. É importante aqui registrar que já ao início houve uma grande adesão, ficando em difícil situação os professores que não compartilhavam da ideia, pois eram considerados "tradicionais". Na medida em que o tempo foi passando, os professores perceberam que esse novo caminho não estava solucionando o problema da alfabetização. Contrariamente, os alunos demoraram mais a se alfabetizar e as dificuldades de leitura aumentaram. Também aqui, houve grande adesão inicial dos professores, mas aos poucos, ao reconhecerem os problemas, não conseguiram fazer transposições didáticas que os superassem, isto é, que organizassem situações e materiais de ensino produtivos e eficientes.

Continuando essa busca no tempo, a partir de bandeira levantada pelos fonoaudiólogos, começaram novas reflexões, passando os professores a perceber a importância do trabalho com consciência linguística. É possível dizer que hoje os professores de anos iniciais estão em processo de revisão das concepções sobre alfabetização.

Embora a ideia de texto venha se ampliando desde os anos 60, a penetração desse ponto de vista junto aos professores começou nos anos 80 , sendo que a definição de gênero textual se estabeleceu bem mais recentemente. $O$ entendimento de que a escola deve trabalhar com a variedade de gêneros e de que eles têm um lugar social ganhou força especialmente com os Parâmetros Curriculares Nacionais. As dificuldades para sua inserção no trabalho escolar no ensino da língua materna provavelmente não decorrem da falta de acolhimento pelos professores, mas da falta de conhecimentos para as transposições didáticas que permitam um estudo linguístico do funcionamento do texto. Isso tem gerado um ensino com gêneros variados, atividades de leitura de suas marcas e atividades de gramática classificatória (usando elementos do texto) dissociadas 
das de leitura. Do mesmo modo aqui o acolhimento dos professores vem sendo muito positivo, não sendo, portanto, essa a causa do insucesso, mas sim provavelmente sua falta de preparo para a produção de materiais de ensino produtivos que dispensem o uso de paradigmas classificatórios.

Como apontado no início deste tópico, esse preparo para a feitura de materiais de ensino exige a passagem, na formação inicial, pela análise de materiais já elaborados, não sendo suficiente desenvolver conhecimentos sobre princípios teóricos $\mathrm{e}$ pedagógicos. À academia cabe essa tarefa, o que provavelmente fortalecerá a disponibilidade para novos paradigmas de ensino da língua materna.

\section{MATERIAIS DE ENSINO DA LEITURA NO PARADIGMA PSICOLINGUÍSTICA E INTERFACES}

A análise realizada no tópico anterior situa na associação teoria e prática uma dificuldade importante para a mudança de paradigma no ensino da língua materna. Essa dificuldade se explica pela necessidade de o futuro professor não só conhecer os fundamentos teóricos e os princípios metodológicos com vistas à elaboração de materiais de ensino, mas também estabelecer contacto com materiais de ensino já elaborados.

Neste tópico são apresentadas definições psicolinguísticas (e interfaces) fundadoras de materiais de ensino da leitura em anos inicias e demonstrações dessas definições que, se incluídas no trabalho de formação inicial dos professores de língua materna pela academia, tanto em curso de Letras quanto em curso de Pedagogia, podem contribuir para a construção de um novo paradigma.

As definições de fundo para materiais de ensino da leitura nos anos iniciais estão vinculadas ao processo de compreensão leitora, ao texto de leitura e aos traços linguísticos desse texto (fonológicos, morfológicos, sintáticos, léxico-semânticos, pragmáticos e textuais), bem como à consciência textual, incluindo a memória. Essas definições são desenvolvidas a seguir, por meio de explicitações e demonstrações.

Para tratamento das definições de compreensão leitora, memória e consciência, a Psicolinguística, eixo do paradigma de ensino da leitura defendido neste artigo (SMITH, 2003), estabelece conexões com as Neurociências, dada a natureza cognitiva processual da compreensão, conforme mostram estudos como os de Dehaene $(2001,2007,2009)$ e Baars (1993). Paralelamente, faz liames com a Psicologia Cognitiva e a Linguística do Texto para as definições de consciência textual, dada sua dupla natureza de tratamento 
do texto e da cognição, como indicam os estudos de Gombert (1992) e Adam (2008). Ao mesmo tempo, a Psicolinguística compõe ligações com a Linguística do Texto para as definições de gênero e tipo textual, cabendo salientar Adam (2008) e Bazerman (2009), e das marcas lingüísticas que os constituem, cabendo referir Charolles (1978) e Hallyday \& Hasan (1976). É nessa rede de interações teóricas que o paradigma de ensino da leitura nos anos iniciais é aqui proposto.

O ensino da leitura supõe um trabalho escolar para o desenvolvimento do processo de compreensão leitora (SPINILLO; MOTA; CORREA, 2010), que conta com a observação das pistas linguísticas deixadas pelo autor no texto e com a ativação dos conhecimentos prévios armazenados pelo leitor na memória declarativa e dos procedimentos de leitura aprendidos e armazenados na memória procedural (EYSENCK \& KEANE, 2007).

Considerando os conhecimentos prévios do leitor, o objetivo da leitura e os traços do gênero textual, a leitura envolve os processos bottom-up ou top-down ou ainda a interação de ambos (SCLIAR-CABRAL, 2008, 2009). Vinculadas ao tipo de processamento estão as estratégias de leitura, sendo fundamentais, pois muito utilizadas algumas delas - skimming, scanning, leitura detalhada, predição, automonitoramento, autoavaliação, autocorreção (SMITH, 2003).

Nesse sentido, materiais de ensino da leitura podem ser organizados de modo que as crianças exercitem diferentes situações e, por decorrência, diferentes processamentos e estratégias (LEFFA, 1996). Aqui estão alguns exemplos que podem exigir diferentes processamentos e estratégias (PEREIRA, 2006, 2009, 2010): leitura de textos para a qual os alunos tenham diferentes níveis de conhecimentos prévios; leitura de textos com diferentes objetivos - para procurar uma informação, para fazer um resumo, para emitir um parecer, para realizar uma análise, para elaborar um comentário, para se distrair, para ter prazer...; leitura de textos pertencentes a diferentes categorias de gêneros - uma propaganda, uma notícia, um conto, um poema, uma curiosidade científica, uma placa, um calendário...

Dando seguimento, eis alguns exemplos de propostas com o objetivo de desenvolver competência no manejo de estratégias de leitura: aproximação rápida de alguns livrinhos de história para inferir o assunto de cada um (skimming); leitura rápida de uma história para identificar o nome de um personagem (scanning); reconto de uma fábula lida (leitura detalhada); antecipação do final de uma anedota (predição); interrupção da leitura de um texto sobre a vida das abelhas, por exemplo, e relato do que 
já compreendeu (automonitoramento); levantamento de hipóteses sobre o próximo quadrinho de uma história com uma sequência deles (predição); julgamento da pertinência das hipóteses levantadas e correção ou confirmação (autoavaliação e autocorreção).

Ensinar o uso de processamentos e estratégias supõe a existência de um material de leitura, isto é, tudo aquilo que traz algo para ler, estruturado nos planos constitutivos da língua, imerso numa situação comunicativa. Neste artigo, é aquele que tem algo escrito podendo apresentar também outros elementos semiológicos, como ocorre no hipertexto. Tal material consiste num texto marcado linguisticamente, escrito com uma intenção para ser lido a partir de um objetivo.

Os estudos sobre gêneros textuais definem os materiais de leitura como textos com função e lugar social. Categorizá-los é algo complexo (BAZERMAN, 2009), uma vez que eles tipificam atitudes e situações sociais e essas estão em continuados movimentos. Isso faz com que estejam constantemente surgindo novos gêneros textuais assim como sendo substituídos ou transformados.

Os gêneros textuais produzidos e lidos em situações de uso possuem marcas linguísticas compatíveis com elas. Uma dessas marcas ou traços está nas sequências dominantes que os estruturam - narrativa, descritiva, expositiva, argumentativa e injuntiva - e que trazem consigo seleções lexicais e gramaticais (ADAM, 2008).

A sequência narrativa é marcada pelo tempo em que os fatos se sucedem da preparação ao fechamento. A descritiva, por sua vez, é predominantemente marcada pelo espaço, constituindo muitas vezes o suporte (cenário) para a narração. A expositiva contribui para o texto, explicitando situações, perspectivas, pontos de vista. A argumentativa tem como elementos-chave a tese e os argumentos, contrapostos à(s) antítese(s), impulsionados pela intenção do autor em persuadir e pelas convicções do leitor e apoiados nas demais sequências. A injuntiva se caracteriza pela interferência sobre o interlocutor, sendo ele chamado por meio de vocativos, pronomes, verbos no imperativo e pontuação gráfica representando a entonação.

Para a construção de materiais de ensino da leitura nos anos iniciais, são gêneros textuais importantes os que usualmente já transitam nas salas de aula, na vida familiar, nas brincadeiras com os amigos, nas horas de lazer. Os gêneros com sequências narrativas, descritivas e expositivas são também pertinentes. Do mesmo modo, os textos mais marcados pela situação comunicativa também são oportunos. São materiais com atividades que exploram os planos linguísticos que os estruturam predominantemente - 
as embalagens, os calendários, as agendas, os cartazes, as tiras, os cartões, os convites, os bilhetes, as cartas, as instruções, as fábulas, as lendas, as parlendas, as cantigas, os poemas, os contos, as adivinhas, as curiosidades, os científicos de assuntos dos diversos componentes curriculares.

Nesse sentido, materiais de ensino da leitura podem ser organizados de modo que as crianças exercitem diferentes gêneros textuais e, por decorrência, suas especificidades no que se refere às marcas linguísticas, como por exemplo, traços fônicos (rimas, aliterações, ritmo) presentes de modo significativo em poemas, quadrinhas, trava-línguas, cantigas e traços pragmáticos bem marcados em cartões, bilhetes, cartas, convites, provérbios, adivinhas.

É importante também que as crianças trabalhem com gêneros de modo a reconhecer a existência dos diversos planos linguísticos, uma vez que assim é a língua constituída. Na cantiga "O cravo brigou com a rosa / debaixo de uma sacada / o cravo saiu ferido / a rosa despedaçada.”, cabe examinar as rimas, o ritmo, a melodia (traços fônicos); o léxico utilizado cravo/rosa, ferido/despedaçada, o significado de saiu, por exemplo (traços léxico-semânticos); as estruturas ferid $+o$, despedaçad $+a$, $s a i+u$ (traços morfológicos); as relações cravo/brigou, cravo/saiu, rosa/despedaçada, cravo/ferido (traços sintáticos); a origem popular da cantiga e o público infantil, considerando a presença no universo de conhecimentos e crenças (traços pragmáticos); a estrutura em versos, o tema se desenvolvendo em um fato central, as repetições lexicais cravo e rosa, a repetição das estruturas sintáticas, os campos semânticos cravo/rosa, cravo/ferido, rosa/despedaçada, a elipse de saiu em a rosa despedaçada (traços textuais).

Os estudos sobre consciência linguística e consciência procedimental definem os materiais de ensino da leitura nos anos iniciais em duas linhas de trabalho - o material de leitura em si como objeto de conhecimento e o processo de leitura realizado pelo leitor como objeto de conhecimento.

São referências importantes as concepções sobre consciência desenvolvidas por Baars e Dehaene. Baars (1993) traz a teoria do espaço global da consciência (global workspace), segundo a qual os conteúdos conscientes estão contidos num espaço global: uma espécie de processador central usado para mediar a comunicação com um conjunto de processadores especializados não conscientes. Dehaene (2001, 2007, 2009) esclarece que, apenas a partir do tempo de 270-300 milissegundos, é possível ver diferença entre o processamento consciente e o inconsciente, ocorrendo a partir do momento em que diferentes áreas do cérebro entram em sincronia. As concepções de Dehaene colaboram 
para explicitar as propriedades da consciência: ativa em sincronia com diversas áreas do cérebro; tem um foco linguístico específico; utiliza informações periféricas a esse foco o contexto; é intencional na busca da análise de algum ponto específico.

A consciência linguística (GOMBERT, 1992) pode focalizar determinado segmento linguístico, considerando sempre o contexto dos demais segmentos. Pode ser categorizada como: consciência fonológica, com foco nos fonemas (consciência fonêmica), nas sílabas (consciência silábica) e nas propriedades entonacionais e rítmicas da língua em uso; consciência morfológica, com ênfase nos limites do vocábulo, na sua estrutura e nos processos derivacionais; consciência sintática, com direcionamento para a frase internamente: limite, estrutura, combinações entre as palavras e pontuação; consciência léxico-semântica, com abrangência do léxico mental (vinculado à memória lexical), do significado (significação básica atribuída pela comunidade, vinculada à memória semântica) e do sentido (construção ad hoc pelo leitor); a consciência pragmática, voltada para a situação de uso da língua, sendo o texto considerado do ponto de vista do enunciador/receptor, do objetivo, do suporte comunicativo, do momento e espaço da comunicação; a consciência textual, com foco nas relações textuais internas e com o contexto - a superestrutura (organização, moldura de formato, componentes constitutivos, sequências dominantes, marcas do gênero), a coesão - elos coesivos de costura das palavras, das frases/versos e dos parágrafos/estrofes (HALLIDAY \& HASAN, 1976) e a coerência (CHAROLLES, 1978) - vínculos internos do conteúdo do texto e com o entorno.

Neste item está apresentado um paradigma para ensino da leitura nos anos iniciais, baseado na Psicolinguística em interface interna com a Linguística do Texto e interface externa com as Neurociências, a Psicologia Cognitiva e a Educação. Esse paradigma envolve compreensão leitora, gêneros textuais e suas marcas linguísticas, consciência linguística em suas diversas dimensões (fonológica, morfológica, sintática, léxico-semântica, pragmática e textual) e seus vínculos com a memória declarativa e a consciência procedimental (memória procedural). $\mathrm{O}$ uso desse ou de qualquer outro paradigma de ensino da leitura nos anos iniciais exige o convívio dos professores em sua formação inicial com a análise e a produção de materiais de ensino, com apoio no paradigma eleito. 


\section{MATERIAIS DE ENSINO DA LEITURA NA FORMAÇÃO INICIAL DO PROFESSOR}

A formação inicial do professor de língua materna cabe, conforme legislação, à academia, seja no curso de Licenciatura em Letras seja no de Pedagogia. Usualmente tanto um como outro possuem disciplinas teóricas, pedagógicas e de aplicação no ensino - o primeiro grupo oportuniza os fundamentos da área de estudos, o segundo propicia vivências de aproximação à prática pedagógica e o terceiro possibilita ao acadêmico a vivência da docência na área de formação.

Com o objetivo de preparação do professor de língua materna, a Licenciatura em Letras dedica-se a isso, enquanto a Pedagogia reserva uma parte muito discreta. Isso faz com que o nível de aprofundamento de conhecimentos sobre a língua seja inegavelmente maior em Letras assim como a abrangência da formação seja maior na Pedagogia. Essa condição traz implicações sobre um ponto importante a respeito de um novo paradigma na formação inicial dos professores - a interface, considerando o quanto é desejável que o ensino da leitura nos anos iniciais seja percebido por diferentes ângulos e seja definido na convergência de diferentes campos do conhecimento. Dadas às características dos cursos, ambos os formatos são, em parte, favoráveis e, em parte, desfavoráveis, pois o trabalho de interface exige ao mesmo tempo conhecimentos verticais e horizontais.

Para benefício da formação inicial do professor de ensino da língua materna é desejável que nos dois cursos a Linguística e a Educação estejam presentes em profundidade, nas correlações internas do próprio campo, nas correlações entre os dois campos e nas correlações com outros campos como as Neurociências e a Psicologia Cognitiva. É desejável também que haja um diálogo mais nítido entre as disciplinas de fundamentos e as pedagógicas, de modo a estreitar as relações entre teoria linguística e teoria de ensino. É do mesmo modo desejável que a Psicolinguística, na medida em que consiste numa ciência de explicitação dos processos de compreensão e produção, tenha um espaço de bastante nitidez nesses cursos. É ainda desejável que a Licenciatura de Letras acolha em seu currículo o desenvolvimento de conhecimentos voltados para os anos iniciais. É finalmente desejável que a prática de ensino seja um momento de profunda convergência dos estudos linguísticos e pedagógicos.

Continuando a reflexão sobre a formação inicial desse professor, é possível constatar que entre as disciplinas de preparação para o ensino há as de tópicos gerais e há as de tópicos específicos. As disciplinas de tópicos gerais, que têm sua importância, 
apresentam, no entanto, grande dificuldade de, mesmo tendo essa natureza, direcionar os conteúdos e os procedimentos de modo a estabelecer vínculos com a formação específica, aqui no caso o ensino da leitura. Isso faz com que os acadêmicos não atribuam a necessária relevância a elas, o que gera um conhecimento isolado dos demais e, por decorrência, da preparação para a docência. As disciplinas de tópicos específicos, que também têm sua importância, tendem a desenvolver atividades de análise crítica de materiais de ensino já reconhecidos como pertencentes a paradigmas pouco produtivos, sendo frequentes, por exemplo, as avaliações de livros didáticos. Isso faz com que os acadêmicos desenvolvam conhecimentos sobre como não devem ser esses materiais de ensino, sem, no entanto, desenvolver conhecimentos sobre como eles devem ser. A situação se agrava na medida em que esses modos reconhecidos como não produtivos são observados pelos acadêmicos em seus professores, especialmente nos das disciplinas teóricas.

Para que o professor aqui em questão tenha uma boa formação inicial, é desejável que as disciplinas de ensino tenham como meta a preparação dos acadêmicos para elaboração de materiais de ensino da leitura. Para isso, cabe às de tópicos gerais, direcionarem sua programação para esses materiais recortando os tópicos gerais com vínculos com os específicos. Às de tópicos específicos, cabe ultrapassar a tendência para a análise crítica do que não deve ser para a análise do que deve ser, priorizando o contacto com materiais de ensino produtivos, por meio de reflexão e produção. É desejável também que os professores dos acadêmicos estejam muito bem informados dessas metas e desenvolvam um ensino no paradigma desejado, o que dará consistência à relação entre discurso pedagógico e prática pedagógica.

Dando seguimento à presente reflexão, é possível perceber que o momento da prática docente (estágio obrigatório) oferece muitas dificuldades aos acadêmicos. Na programação das aulas de ensino da leitura, os acadêmicos evidenciam dificuldades para selecionar textos, definir os conteúdos de leitura e organizar a sequência didática, enfim para organizar os materiais de ensino. Falta densidade aos conceitos, especialmente aos de leitura, compreensão, processamento, gênero textual, sequência textual, marcas linguísticas, coerência e coesão, gerando impossibilidades de transposição para a prática e, consequentemente, de transformação em materiais de ensino da leitura. Paralelamente, falta-lhes guarida teórica e metodológica na escola para um novo paradigma - ao contrário, são solicitados pelos professores, pelos alunos e pelos familiares para o uso de paradigmas criticados na academia. Nesse impasse, buscam 
saída nos materiais dos professores, nos livros didáticos e nas suas próprias experiências como alunos do tempo de escola. Nessa conjuntura, o docente orientador do estágio faz um curso relâmpago desenvolvendo conceitos, discorrendo sobre metodologia e orientando a elaboração e a aplicação de materiais de ensino da leitura.

Para que a formação inicial do professor de ensino da língua materna seja produtiva, é desejável a convergência entre o papel que o estagiário deve desempenhar e os preparos prévios por meio das diversas disciplinas, de modo que a feitura dos materiais de ensino da leitura seja uma decorrência natural. É também desejável que a academia desenvolva projeto prévio de interação com a escola no que se refere ao paradigma de ensino da leitura, de modo que ele seja um querer duplo - da academia e da escola. É ainda desejável que o momento da prática docente oportunize o intercâmbio de conhecimentos e a adesão da escola a esse paradigma de materiais de ensino da leitura para além da situação de um estágio docente.

\section{COMENTÁRIOS FINAIS}

O presente ensaio desenvolve-se em três itens. No primeiro deles é feita uma análise da formação inicial dos professores de língua materna, salientando os materiais de ensino da leitura nos anos iniciais. No segundo, é fundamentado e demonstrado um paradigma de ensino que contempla esses materiais, baseado na Psicolinguística em interface interna com a Linguística do Texto e em interface externa com as Neurociências, a Psicologia Cognitiva e a Educação. Esse paradigma está apoiado nos conceitos de leitura, compreensão, processamento, gêneros e sequências textuais, consciência linguística/memória declarativa e consciência procedimental/memória procedural. No terceiro, desenvolvem-se análises e reflexões sobre o paradigma dos materiais de ensino da leitura, estabelecendo relações com um paradigma de atuação da academia. Nesse item, examinam-se procedimentos desejáveis da academia para que esses paradigmas se viabilizem.

As informações, as análises, as reflexões e as proposições apresentadas possibilitam alguns encaminhamentos finais. Nesse quadro de dificuldades para a mudança de paradigma dos professores de ensino de língua materna, a formação inicial tem uma grande responsabilidade. Um tópico que está alojado nesse paradigma é o dos materiais de ensino da leitura nos anos iniciais. Dadas às movimentações que a contemporaneidade vive, a hora é a das interfaces, o que cabe adotar para a construção 
desse paradigma. Como a formação inicial dos professores é um lugar e um momento determinantes para o seu preparo na direção de um paradigma produtivo, cabe à academia refletir sobre o seu próprio paradigma no que se refere aos conhecimentos que disponibiliza aos professores em formação e aos procedimentos que utiliza, de modo a contribuir de modo mais significativo para a construção desse paradigma de materiais de ensino da leitura nos anos iniciais, em que a Psicolinguística na interface interna com a Linguística do Texto e na interface externa com as Neurociências, a Psicologia Cognitiva e a Educação tem um papel especialmente relevante.

No fechamento destas reflexões, é importante ampliar as considerações e os comentários aqui direcionados aos anos iniciais para os demais níveis de escolaridade, se o desejo é contribuir para a produtividade do sistema de ensino de língua materna brasileiro.

\section{REFERÊNCIAS}

ADAM, Jeam-Michel. A Linguística: introdução à análise textual dos discursos. São Paulo: Cortez, 2008.

BAARS, Bernard J.. A cognitive theory of consciousness. Cambridge: Cambridge Univ., 1993.

BAZERMAN, Charles. Dionísio, A. P.; Hoffnagel, J. C. (Orgs.). Gêneros textuais, tipificação e interação. São Paulo: Cortez, 2009.

CHAROLLES, Michel. Introduction aux problèmes de la cohérence des textes. Langue Française. Paris: Larousse, n.38, p. 7-41, 1978.

DEHAENE, Stanislas. The cognitive neuroscience of consciouness. Cambridge, Massachussetts: The MIT Press, 2001.

DEHAENE, Stanislas. Les neurones de la lecture. Paris: Odile Jacob, 2007.

DEHAENE, Stanislas. Signatures of consciousness - a talk by Stanislas Dehaene. Edge in Paris. Entrevista concedida a Edge Foundation, Inc., 2009. Disponível em: http://www.edge.org/3rd_culture/dehaene09/dehaene09_index.html.

EYSENCK, M.W.; KEANE, M.T.. Manual de Psicologia Cognitiva. Porto Alegre: Artmed, 2007.

GOMBERT, Jean Émile. Metalinguistic development. Chicago: The University of Chicago Press, 1992.

HALLIDAY, M. A. K. \& HASAN, R.. Cohesion in English. London: Longman, 1976. 
LEFFA, Vilson J.. Aspectos da leitura: uma perspectiva psicolinguística. Porto Alegre: Sagra-Luzzatto, 1996.

PEREIRA, Vera Wannmacher. Computer-based learning of reading and writing im Elementary School. In: Cestari, M. L.; Massagero, E.; Tonnessen, E. Networking cultures: Brazilian-Norwegian dialogues on education and culture. Norway: Portal Ebooks, p. 107-18, 2006.

PEREIRA, Vera Wannmacher. Estratégia de predição leitora nas séries iniciais: resultados de pesquisa e aplicação no ensino. Acta Scientiarum: language and culture, v. 3, n. 2, p.133-38, 2009.

PEREIRA, Vera Wannmacher. (2010). Aprendizado da leitura e consciência linguística. Anais do IX Encontro do CELSUL. Palhoça, SC. Universidade do Sul de Santa Catarina, p. 1-11.

SCLIAR-CABRAL, Leonor. Processamento bottom-up na leitura. Veredas on-line, p. 24-33, 2008. Disponível em http://www.ufjf.br/revistaveredas/files/2009/12/artigo02.pdf.

SCLIAR-CABRAL, Leonor. Processamento da leitura: recentes avanços das neurociências. In: PEREIRA, Vera Wannmacher; COSTA, Jorge Campos da (Orgs.). Linguagem e cognição: relações interdisciplinares. Porto Alegre: EDIPUCRS, p. 4960, 2009.

SMITH, Frank. Compreendendo a leitura: uma análise psicolinguística da leitura e do aprender a ler. Porto Alegre: Artes Médicas, 2003.

SPINILLO, A. G.; MOTA, M. M. P. E.; CORREA, J.. Consciência metalinguística e compreensão da leitura: diferentes facetas de uma relação complexa. Educar em Revista, n. 38, p. 157-171, 2010.

\section{A AUTORA}

Vera Wannmacher Pereira é professora titular do Programa de Pós-graduação em Letras da PUCRS. Possui Doutorado em Letras e Pós-doutorado em Psicolinguística. É bolsista de Produtividade do CNPq. Desenvolve, com base na Psicolinguística e suas Interfaces com a Educação, a Computação e as Neurociências, pesquisas vinculadas a ensino e extensão sobre compreensão e processamento da leitura e da escrita. Coordena o Centro de Referência para o Desenvolvimento da Linguagem - CELIN da Faculdade de Letras da PUCRS.

E-mail: vpereira@pucrs.br 